\title{
Die Produktarchitektur als zentrales Konzept in der Produktentwicklung
}

David Inkermann ${ }^{1}$, Michael Hanna ${ }^{2}$, Timo Richter ${ }^{1}$, Nadine Wortmann $^{2}$, Thomas Vietor ${ }^{1}$, Dieter Krause ${ }^{2}$

${ }^{1}$ Institute for Engineering Design (IK),

Technische Universität Braunschweig, Germany

2 Institute of Product Development and Mechanical Engineering Design (PKT), Hamburg University of Technology (TUHH), Germany

\section{Abstract}

As a result of the development process every technical system has a specific product architecture. This architecture is based on the intended purpose of the product as well as technical-functional, product strategic and live cycle requirements and impacts essential properties like mass, assembly or changeability of single components. In order to elaborate and adapt the product structure there are numerous strategies, methods and design principles that are using the concept of the product architecture. Here the product architecture is understood as the coupling of the functional and physical description of the product. To clarify the relevance of a goal-oriented design of the product architecture in this contribution we align and systemize goals, strategies, methods and design principles.

Keywords: Product Architecture, Modular Product Structures, Methods and Strategies for Product Architecture Design 


\section{$1 \quad$ Einleitung}

Als Ergebnis des Entwicklungsprozesses weist jedes technische System eine spezifische Produktarchitektur auf. Die realisierte Produktarchitektur basiert auf dem geforderten Produktzweck sowie technisch-funktionalen, produktstrategischen und lebenszyklusbezogenen Anforderungen und bestimmt maßgeblich Eigenschaften wie Gewicht, Montierbarkeit oder Austauschbarkeit einzelner Komponenten. Gleichzeitig hat sie wesentlichen Einfluss auf die Handhabung von Variantenvielfalt, weshalb häufig modulare Produktstrukturstrategien angewendet werden [1]. Für die gezielte Erarbeitung neuer und die Anpassung bestehender Produktstrukturen existiert eine Vielzahl von Strategien, Methoden und Konstruktionsprinzipien, die auf das Konzept der Produktarchitektur zurückgreifen. Die Produktarchitektur wird hierbei als Verknüpfung der funktionalen und physischen Beschreibung technischer Systeme verstanden [2, 3]. Bestehende Arbeiten verdeutlichen die Vielzahl und deutlichen Unterschiede von Betrachtungsschwerpunkten, Zielsetzungen und Strategien, die für die Gestaltung der Produktarchitektur vorgeschlagen werden. Um ein Verständnis für die Bedeutung der zielgerichteten Gestaltung der Produktarchitektur zu vermitteln und Zielstellungen sowie Methoden bereitzustellen, werden in diesem Beitrag Zielstellungen, Strategien und Methoden abgeglichen und systematisiert. Ziel ist es, einerseits zentrale Begriffe zu vereinheitlichen und andererseits deren Wechselwirkungen für die systematische Gestaltung der Produktarchitektur zu definieren. Das vorgeschlagene Begriffssystem sowie die aufgezeigten Zielstellungen, Bauweisen, Strategien und Methoden sollen das Konzept der Produktarchitektur - als zentralen Stellhebel zur Erreichung unterschiedlicher Ziele - in der Produktentstehung für Ausbildung und Praxis nutzbar machen.

Im nachfolgenden Abschnitt wird zunächst der Begriff Produktarchitektur definiert und der Zusammenhang zwischen Zielstellungen, Bauweisen, Strategien und Methoden bei der Gestaltung der Produktarchitektur aufgezeigt. In den Abschnitten 3 bis 6 werden ausgewählte Zielstellungen, Bauweisen, Strategien und Methoden für die Produktarchitekturgestaltung beschrieben. Abschließend werden in Abschnitt 7 zukünftige Forschungsfelder im Themenfeld der Produktarchitekturgestaltung skizziert.

\section{Produktarchitektur - Zentrale Definitionen und Zusammenhänge}

Die zahlreichen bestehenden Definitionen in der Literatur verdeutlichen die unterschiedlichen Betrachtungsschwerpunkte bei der Gestaltung der Produktarchitektur. In diesem Abschnitt wird das Konzept der Produktarchitektur definiert und zentrale Aspekte und Handlungsfelder für die zielgerichtete Produktarchitekturgestaltung beschrieben. 


\subsection{Definition der Produktarchitektur}

Für die unterschiedlichen Analyse- und Synthesetätigkeiten während der Produktentwicklung werden verschiedene Beschreibungen des Produkts zugrunde gelegt. Einerseits wird häufig eine lösungsneutrale, zweckangebende funktionale Beschreibung des Produkts - die Funktionsstruktur - erarbeitet, um Zweck und gefordertes Verhalten des zu entwickelnden Systems anzugeben. Andererseits erfolgt eine physische Definition des Produkts in Form der Produktstruktur. Durch die Produktarchitektur wird die funktionale und physische Beschreibung des Produkts verknüpft und damit der Übergang zwischen beiden Sichtweisen während der Entwicklung unterstützt. In Anlehnung an [4, 2] ist demnach folgende Definition zielführend: Die Produktarchitektur fasst die Produktstruktur als physischen Aufbau und die Funktionsstruktur als funktionale Beschreibung eines Produkts zusammen und stellt deren Elemente miteinander in Beziehung. In Bild 1 ist das Konzept der Produktarchitektur als Zuordnung von Teilfunktionen zu Komponenten eines Produkts sowie deren hierarchische Gliederung innerhalb der Funktions- und Produktstruktur dargestellt.

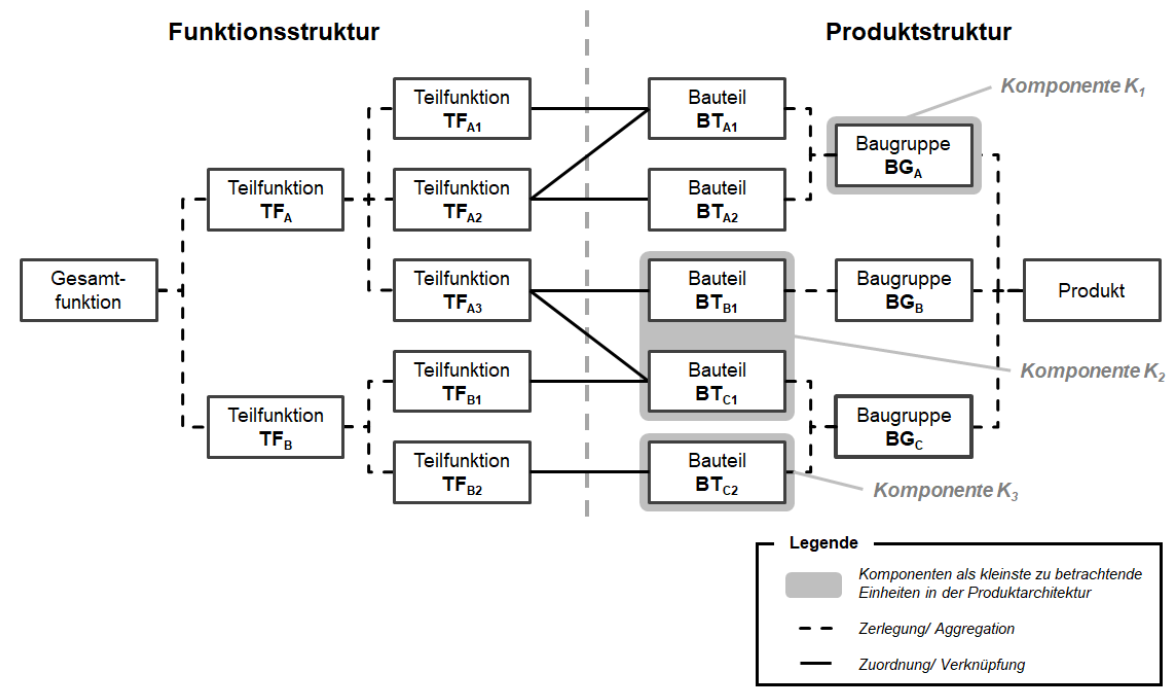

Bild 1: Schematische Darstellung der Produktarchitektur als Verknüpfung der Funktions- und der Produktstruktur [5]

In Abhängigkeit der vorliegenden Analyse- oder Syntheseaktivität muss die geeignete Betrachtungsebene der Produktarchitektur (Gesamtproduktebene, Ebene einzelner Teilsysteme und -funktionen) definiert werden. Hierfür kann die Funktionsstruktur des Produkts hierarchisch mit den Aggregationsebenen 
Gesamtfunktion, Teilfunktionen (vgl. TFA in Bild 1) sowie untergeordneten Teilfunktionen (vgl. $\mathrm{TF}_{\mathrm{A} 1}$ ) gegliedert werden. Ziel ist es hierbei, die geforderten Funktionen und ihre Detaillierungen abzubilden. Die Produktstruktur beschreibt die physischen Bestandteile und ihre hierarchische Gliederung in Produkt, Baugruppen ( $\mathrm{vgl} . \mathrm{BG}_{\mathrm{A}}$ ) und Bauteilen ( $\mathrm{vgl}$. $\mathrm{BT}_{\mathrm{A} 1}$ ) und bildet deren physischen Beziehungen zueinander $a b$. Oftmals erfolgt auch eine Gliederung der Produktstruktur in Komponenten, die spezifisch für den vorliegenden Anwendungsfall definiert werden. Je nach Betrachtungsebene können Komponenten einzelne Bauteile ( $\mathrm{vgl} . \mathrm{K}_{3}$ ) sein oder mehrere Bauteile ( $\mathrm{vgl} . \mathrm{K}_{2}$ ) umfassen, die für diesen spezifischen Anwendungsfall die kleinsten zu betrachtenden Einheiten der Produktstruktur bilden. Auch eine gesamte Baugruppe (vgl. BG $\mathrm{B}_{\mathrm{A}}$ ) kann als eine Komponente ( $\mathrm{vgl}$. $\mathrm{K}_{1}$ ) angesehen werden. In den folgenden Abschnitten wird vereinfachend nur von Komponenten gesprochen, die entsprechend dieser Definition Bauteile oder Baugruppen umfassen können.

\subsection{Zusammenhänge bei der Gestaltung der Produktarchitektur}

Die zielgerichtete Analyse und Gestaltung der Produktarchitektur setzt die Berücksichtigung unterschiedlicher Aspekte und Handlungsfelder voraus. Durch die Unterscheidung von Zielstellungen, Strategien, Bauweisen und Methoden stellt der Beitrag ein Begriffssystem vor, dass eine strukturierte Gestaltung der Produktarchitektur unterstützt und die Systematisierung bestehender Ansätze erlaubt. In Bild 2 sind die zentralen Betrachtungsebenen und Begriffe sowie ihre Wechselwirkungen zusammenfassend dargestellt. Ausgangspunkt für die Gestaltung der Produktarchitektur sind demnach Zielstellungen, die angestrebte Verbesserungen von Produkteigenschaften oder Prozessen angeben. Diese Zielstellungen sind Grundlage für die Auswahl von Bauweisen sowie Methoden und werden durch Strategien unterstützt. Bauweisen beruhen auf etablierten Konstruktionsprinzipien wie der Vereinigung mehrerer Komponenten mit gleichen oder unterschiedlichen Funktionen zu einer neuen Komponente (Integralbauweise). Sie geben damit operativ umsetzbare Gestaltungshinweise für die Produktstruktur. Methoden zur Produktarchitekturgestaltung können die Auswahl geeigneter Bauweisen und die Umsetzung von Strategien unterstützen. Die Auswahl geeigneter Methoden beruht auf der Festlegung spezifischer Zielstellungen. Strategien für die Gestaltung der Produktarchitektur stellen allgemeine und (unternehmensspezifisch) etablierte Lösungsansätze für die Produktstrukturierung dar. Im Vergleich zu Bauweisen sind Strategien, wie die Plattformstrategie, deutlich weitreichender und berücksichtigen technische, strategische und organisatorische Aspekte. Die im Beitrag eingeführten Strategien gehen insbesondere auf die Herausforderungen der Variantenvielfalt und dem verbundenen Zielkonflikt zwischen Standardisierung und Differenzierung ein. 


\section{Zielstellungen}

Zielstellungen beschreiben mögliche Auswirkungen der gewählten Produktarchitektur auf Produkte und Prozesse sowohl aus Sicht eines Unternehmens als auch eines Kunden. Zielstellungen sind Grundlage für die Auswahl von Bauweisen, Strategien und Methoden für die Gestaltung der Produktarchitektur.

Beispiel: Vermeidung und Reduzierung interner Variantenvielfalt

\section{Strategien}

Strategien beschreiben allgemein anwendbare und etablierte Änsätze zur Produktstrukturierung vor dem Hintergrund spezifischer Zielstellungen. Neben technischfunktionalen Aspekten werden hierbei produktstrategische und organisatorische Aspekte berücksichtigt. Beispiele: Plattformstrategie, Baureihenstrategie

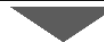

\section{$+$}

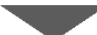

Gestaltung der Produktarchitektur

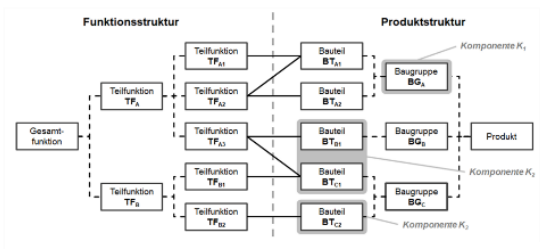

\section{Bauweisen}

Bauweisen beschreiben wiederkehrende, häufig angewendete

Produktstrukturen und diesen zugrundeliegende Konstruktionsprinzipien Bauweisen enthalten operative Gestaltungs- 1 Umsetzungshinweise.

Beispiele:

integralbauweise Modulbauweise

\section{Methoden}

Methoden (für die Gestaltung der Produktarchitektur) beschreiben ein planmäßiges Vorgehen und spezifische Hilfsmittel für die Umsetzung von Strategien und Realisierung von Bauweisen.

Beispiel: Modular Function Deployment, Methode der eintelligen Maschine

Bild 2: Zentrale Begriffe zur Gestaltung der Produktarchitektur.

Die Analyse und Gestaltung der Produktarchitektur erfolgt im Hinblick auf unterschiedliche Zielstellungen. Diese Zielstellungen geben beispielsweise geforderte Produkteigenschaften während der Nutzungsphase sowie weiterer Produktlebensphasen innerhalb der Produktentstehung, wie der Absicherung oder Produktion, an. Als Grundlage für die Auswahl von Strategien, Methoden und Bauweisen ist es daher zweckmäßig, die spezifischen Zielstellungen der vorliegenden Entwicklungsaufgabe explizit zu klären. Da zugrunde gelegte Bauweisen zur Realisierung einer Produktstruktur zu verschiedenen Zielstellungen führen, sind neben den unmittelbar verfolgten Zielen auch negative Auswirkungen der gewählten Bauweise zu beurteilen. In diesem Abschnitt wird ein Überblick über mögliche Zielstellungen für die Analyse und Gestaltung der Produktarchitektur gegeben. Die Übersicht beschränkt sich auf eine Auswahl in der Literatur genannter Zielstellungen, die in der dargestellten Form keinen Anspruch auf Vollständigkeit hat, vgl. beispielsweise $[4,6,7]$. Die skizzierten Zielstellungen 
werden nachfolgend anhand der Sichtweise des Unternehmens und des Kunden sowie der Unterscheidung zwischen der Produkt- und Prozessperspektive differenziert, siehe Quadranten in Bild 3. In realen Entwicklungssituationen tritt in der Regel eine Kombination mehrerer Zielstellungen auf, die untereinander priorisiert werden müssen. Unabhängig von der Entwicklungsaufgabe sind immer die übergeordneten Ziele Zeit, Qualität, Kosten und Flexibilität zu berücksichtigen. Die in Bild 3 dargestellten Quadranten legen exemplarische Schwerpunkte für die Analyse und Gestaltung der Produktarchitektur fest, indem sie einzelne Sichten auf Produkte oder Prozesse aus Kunden- oder Unternehmensperspektive hervorheben.

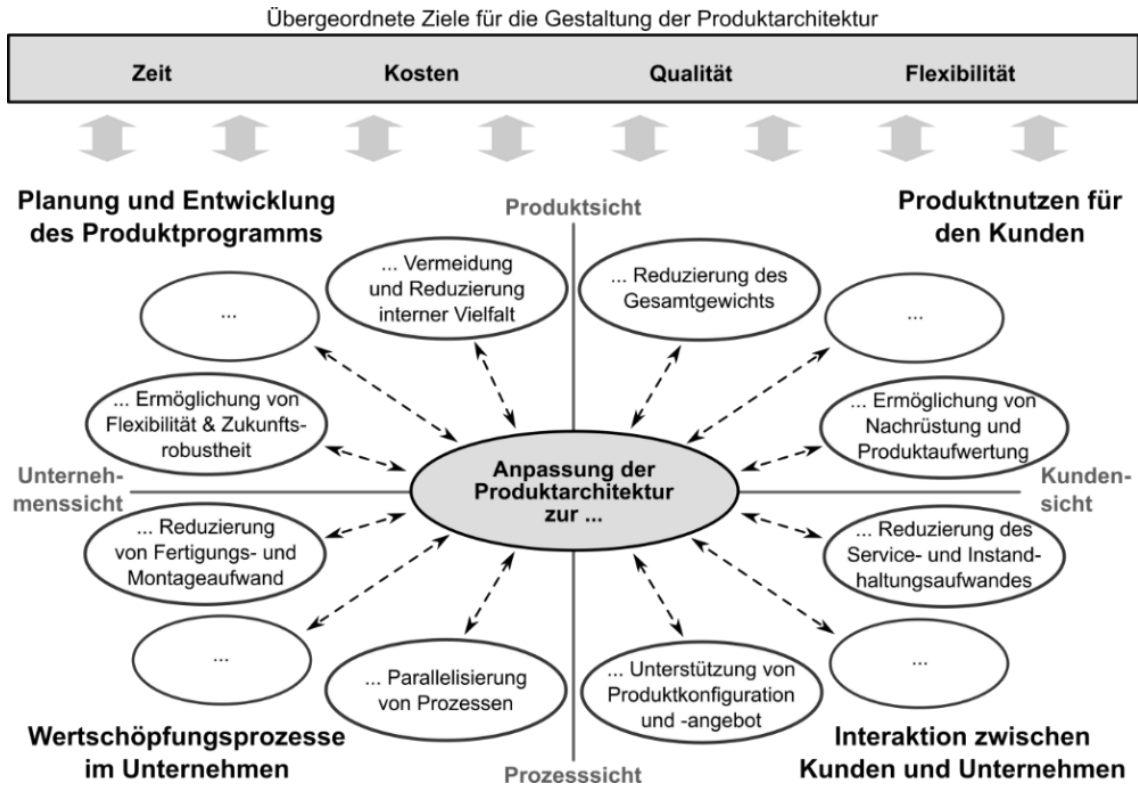

Bild 3: Exemplarische Zielstellungen für die Gestaltung und Anpassung der Produktarchitektur [5].

Der Quadrant Planung und Entwicklung des Produktprogramms zeigt produktbezogene Zielstellungen der Produktarchitektur aus Sicht des Unternehmens auf. Ziel ist es hierbei, Kundenwünsche durch Integration technologischer Neuerungen und Verbesserungen beziehungsweise Differenzierung funktionaler, ästhetischer oder wirtschaftlicher Produkteigenschaften zu erfüllen. Zentrale Herausforderung ist hierbei häufig die Vermeidung und Reduzierung interner Vielfalt von Komponenten aufgrund der geforderten großen externen Produktvielfalt [2]. Die zielgerichtete Gestaltung der Produktarchitektur kann dazu 
beitragen, höhere Kosten zu vermeiden, in dem die interne Variantenvielfalt durch Modulbauweisen reduziert wird [8]. Der Quadrant Wertschöpfungsprozesse im Unternehmen stellt die Zielstellungen im Hinblick auf unternehmensinterne und -übergreifende Prozesse dar, um die Entwicklung, Herstellung und Montage des Produkts kosten- und zeiteffizient zu gestalten. Insbesondere bei der Entwicklung von Produkten mit Lösungselementen unterschiedlicher Entwicklungsdomänen, wie mechatronischen Produkten, oder bei standortübergreifenden Entwicklungsprojekten ist es zweckmäßig, Entwicklungs-, Produktions- und Testaufgaben durch eine geeignete Produktstruktur zu gliedern. Durch Parallelisierung von Prozessen können einzelnen Komponenten weitgehend unabhängig voneinander entwickelt, hergestellt und getestet werden, bevor sie zu einem Gesamtsystem integriert werden. Durch eine modulare Bauweise können hierbei Zeitersparnisse im Produktentstehungsprozess erzielt werden [9]. Der Quadrant Produktnutzen für den Kunden hebt die kundenspezifische Sicht auf das Produkt hervor. Im Fokus stehen dabei häufig die Erfüllung kundenindividueller Anforderungen oder das Angebot zusätzlicher Produktfunktionen während der Nutzungsphase. Beispielsweise können durch die Gestaltung der Produktarchitektur und die Anwendung einer geeigneten Bauweise einzelne, für den Nutzer maßgebliche, Beschaffenheits- und Gebrauchseigenschaften wie Gewicht, Robustheit oder Anpassbarkeit, beeinflusst werden. Eine Integral- oder Verbundbauweise kann beispielsweise maßgeblich zur Reduzierung des Gesamtgewichts des Produkts beitragen. Der vierte Quadrant Interaktion zwischen Kunden und Unternehmen zeigt mögliche Zielstellungen bei der Anpassung der Produktarchitektur mit Auswirkungen auf die Prozessgestaltung aus Sicht des Kunden auf. Eine exemplarische Zielstellung ist die Unterstützung von Produktkonfiguration und -angebot. Durch die Produktarchitektur wird einerseits der Umfang der für den Kunden möglichen Individualisierung des Produkts definiert. Andererseits wird die Produktkonfiguration maßgeblich beeinflusst, indem beispielsweise Konfigurations- und Kalkulationsmöglichkeiten vorgegeben werden, die potentielle Kunden als Entscheidungsgrundlage heranziehen können [10].

Die skizzierten Quadranten zeigen übergeordnete Zielbereiche für die Gestaltung der Produktarchitektur auf. Die beschriebenen Zielstellungen und deren Einordnung in diese Quadranten geben konkrete Beispiele für Ausgangspunkte bei der Gestaltung der Produktarchitektur an. Hervorzuheben ist, dass einzelne Strategien und Bauweisen in der Regel zu Wechselwirkungen zwischen den Zielstellungen führen, weshalb eine Priorisierung der Zielstellungen für die vorliegende Entwicklungsaufgabe erforderlich ist. 
Ergebnis der gezielten Gestaltung der Produktarchitektur ist eine Bauweise, die technische und/oder wirtschaftliche Anforderungen bestmöglich erfüllt. Bauweisen geben wiederkehrende, häufig angewendete Produktstrukturen und diesen zugrundeliegende Konstruktionsprinzipien an. Sie enthalten damit operative Gestaltungs- und Umsetzungshinweise für die Realisierung der Produktstruktur ausgehend von spezifischen Zielsetzungen. Mit der Integral-, Differential-, Verbund- und der Modulbauweise sowie der integrierenden und der Multifunktionalbauweise werden in Tabelle 1 etablierte Bauweisen beschrieben. Die charakteristischen Eigenschaften dieser Bauweisen unterstützen die Erreichung unterschiedlicher Zielstellungen bei der Gestaltung der Produktarchitektur. Beispielsweise sind die Vorteile der Integral- und Differentialbauweisen in der Regel stark stückzahlabhängig. Vereinfachend kann daher angenommen werden, dass die Vorteile der Differentialbauweise, insbesondere bei Produkten in Einzelfertigung oder Kleinserien, erzielt werden. Bei großen Stückzahlen hingegen ist die Integralbauweise häufig besonders geeignet, da die Komponenten in Integralbauweise mithilfe geeigneter Fertigungsverfahren, wie Druckguss oder Spritzguss, kostengünstig hergestellt werden können. Aus diesen Eigenschaften kann beispielsweise die grundsätzliche Stückzahleignung beider Bauweisen abgeleitet werden, vgl. Bild 3. Die einzelnen Verläufe zeigen qualitativ die für die jeweilige Stückzahl vorgeschlagene Bauweise auf.

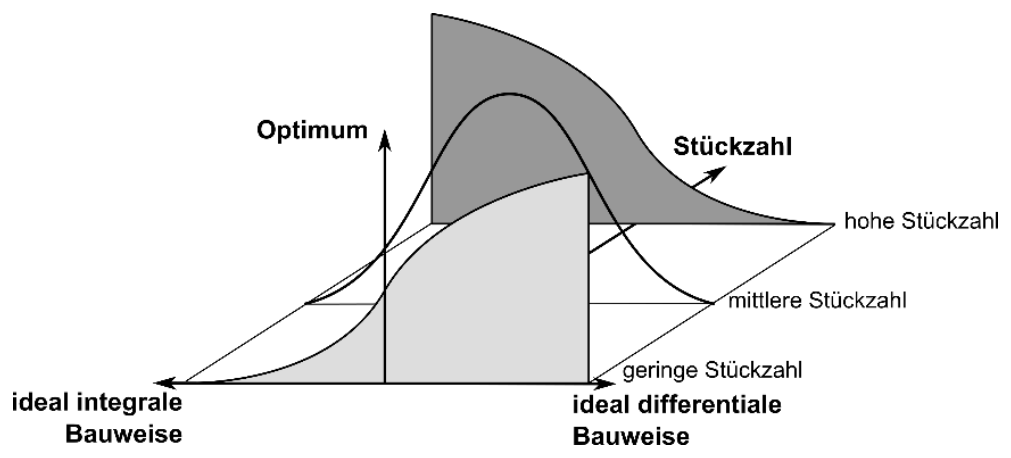

Bild 4: Grundsätzliche Stückzahleignung von Integral- und Differentialbauweise, in Anlehnung an [10].

In der Praxis zeigt sich, dass Bauweisen häufig miteinander kombiniert werden. Die Kombination erfolgt auch durch Anwendung der Bauweisen auf unterschiedlichen Aggregationsebenen. Beispielsweise kann ein Produkt in Modulbauweise ausgeführt und einzelne Module des Produktes in Integralbauweise realisiert werden. Somit werden die Vorteile beider Bauweisen genutzt. 
Tabelle 1: Übersicht typischer Bauweisen zur Produktarchitekturgestaltung

\begin{tabular}{|c|c|}
\hline Bauweise & Konstruktionsprinzip und Zielsetzung \\
\hline $\begin{array}{l}\text { Integral- } \\
\text { bauweise }\end{array}$ & $\begin{array}{l}\text { Bei der Integralbauweise wird das Konstruktionsprinzip verfolgt, } \\
\text { mehrere Komponenten mit gleichen oder unterschiedlichen Funktio- } \\
\text { nen zu einer neuen Komponente mit gleichbleibendem Funktionsum- } \\
\text { fang zu vereinen. Ziel der Integralbauweise ist die Verbesserung ein- } \\
\text { zelner Produkteigenschaften, wie Gewicht, Steifigkeit oder Robust- } \\
\text { heit, durch den Entfall von Schnittstellen. Weiterhin können Kosten } \\
\text { reduziert werden, indem beispielsweise Montageaufwände verringert } \\
\text { oder Fertigungsmittel eingespart werden. In Anlehnung an [11, 12]. }\end{array}$ \\
\hline $\begin{array}{l}\text { Differential- } \\
\text { bauweise }\end{array}$ & $\begin{array}{l}\text { Bei der Differentialbauweise wird das Konstruktionsprinzip verfolgt, } \\
\text { ein Produkt oder eine Komponente bei gleichbleibendem Funktions- } \\
\text { umfang in mehrere neue Komponenten zu zergliedern. Durch die } \\
\text { Zergliederung können Produkteigenschaften verbessert werden, in- } \\
\text { dem die Materialauswahl und Bauteilgestaltung der einzelnen Kom- } \\
\text { ponenten für die vorliegenden Anforderungen angepasst werden. Zu- } \\
\text { dem kann die Differentialbauweise dazu beitragen, den Anteil an } \\
\text { Gleichteilen oder Wiederholteilen innerhalb eines Produkts zu erhö- } \\
\text { hen. In Anlehnung an [11]. }\end{array}$ \\
\hline $\begin{array}{l}\text { Moc } \\
\text { bau }\end{array}$ & $\begin{array}{l}\text { Die Modulbauweise verfolgt das Konstruktionsprinzip, Produkte in } \\
\text { Module zu gliedern, die untereinander möglichst stark entkoppelt } \\
\text { sind, während die Komponenten innerhalb der einzelnen Module } \\
\text { starke Beziehungen aufweisen. Durch die Kombination der Module } \\
\text { wird eine effiziente Bildung von Produktvarianten ermöglicht. Ziel der } \\
\text { Modulbauweise ist es, Module zu definieren die eine effiziente Ent- } \\
\text { wicklung und Herstellung mehrerer Produktvarianten einer Produkt- } \\
\text { familie unter Berücksichtigung technisch-funktionaler und produkt- } \\
\text { strategischen Kriterien ermöglichen. In Anlehnung an [2]. }\end{array}$ \\
\hline $\begin{array}{l}\text { Verbu } \\
\text { bauw }\end{array}$ & $\begin{array}{l}\text { Bei der Verbundbauweise wird das Konstruktionsprinzip verfolgt, } \\
\text { durch unterschiedliche Fertigungsverfahren erzeugte Komponenten } \\
\text { oder Komponenten unterschiedlicher Werkstoffe unlösbar miteinan- } \\
\text { der zu verbinden. Die Verbundbauweise zielt somit darauf ab, Eigen- } \\
\text { schaften unterschiedlicher Materialien gezielt in einer Komponente zu } \\
\text { vereinen. In Anlehnung an [11]. }\end{array}$ \\
\hline $\begin{array}{l}\text { Integrie- } \\
\text { rende } \\
\text { Bauweise }\end{array}$ & $\begin{array}{l}\text { Die integrierende Bauweise stellt eine Kombination der Konstrukti- } \\
\text { onsprinzipien der Integral- und Differentialbauweise dar. Es werden } \\
\text { gezielt Schnittstellen zwischen Komponenten eingefügt, um beispiels- } \\
\text { weise Rissausbreitung, Korrosion oder Kerbprobleme zu vermeiden. } \\
\text { Die Anzahl der Trennungen je Komponente auf ein sinnvolles Maß } \\
\text { begrenzt, um eine gute Austauschbarkeit zu gewährleisten und } \\
\text { Leichtbauanforderungen zu erfüllen. In Anlehnung an [13]. }\end{array}$ \\
\hline
\end{tabular}


Tabelle 1: Übersicht typischer Bauweisen zur Produktarchitekturgestaltung (Fortsetzung)

\begin{tabular}{|l|l|}
\hline Bauweise & Konstruktionsprinzip und Zielsetzung \\
\hline $\begin{array}{l}\text { Multifunktio- } \\
\text { nalbauweise }\end{array}$ & $\begin{array}{l}\text { Mit dem Konstruktionsprinzip der Multifunktionalbauweise wird das } \\
\text { Ziel verfolgt, zusätzliche Funktionen durch eine Komponente oder } \\
\text { ein Bauteil zu realisieren, indem bestehende geometrische oder } \\
\text { stoffliche Eigenschaften einer Komponente genutzt werden. Es wird } \\
\text { eine Funktionsintegration realisiert, indem entweder der Gesamt- } \\
\text { funktionsumfang des Produkts durch zusätzliche Funktionen, soge- } \\
\text { nannte Gratisfunktionen, erhöht wird oder der Gesamtfunktionsum- } \\
\text { fang bei verringerter Anzahl an Komponenten erhalten bleibt. In } \\
\text { Anlehnung an [14]. }\end{array}$ \\
\hline
\end{tabular}

Aufgrund der unterschiedlichen Konstruktionsprinzipien tragen die beschriebenen Bauweisen zur Erreichung verschiedener Zielstellungen bei. Die Auswahl und Anwendung der Bauweisen erfolgt in der Regel ausgehend von bestehenden Produktstrukturen beispielweise vorhandener Produkte und unter Berücksichtigung der spezifizierten Zielstellungen.

\section{Strategien für die Gestaltung der Produktarchitektur}

Eine der wesentlichen Zielstellungen der Gestaltung der Produktarchitektur ist die Berücksichtigung und Realisierung der Produktvielfalt. Dafür werden in Unternehmen Strategien definiert, die Vorgaben für die Produktstrukturierung festlegen. Der Fokus liegt damit auf der Gestaltung der Produktstruktur (physische Sicht). Die Auswahl einer Strategie zur Produktstrukturierung kann sich auf eine Vielzahl von Zielstellungen des Unternehmens auswirken, da unterschiedliche Produktlebensphasen der Produktentstehung und -nutzung eng mit der Produktstruktur verknüpft sind. Strategien für die Gestaltung der Produktarchitektur gelten dabei häufig als unternehmensweite Vorgaben bezüglich der Ausführung der Produktstrukturen zur Erreichung spezifischer Unternehmensziele. Sie sind produktübergreifend bis hin zum gesamten Produktprogramm gültig und haben eine mittel- bis langfristige Ausrichtung, die eine oder mehrere Produktgenerationen umfasst [2]. Die einzelnen Strategien wirken sich auch auf die Ausgestaltung der Bauweise aus. Nachfolgend wird ein Überblick über etablierte Strategien für die Gestaltung der Produktarchitektur gegeben. Etablierte Strategien für die Reduzierung der internen Vielfalt sind die Gleichmodul, Modulbaukasten- und Plattformstrategie, vgl. Bild 4. Diese Strategien spannen den Lösungsraum modularer Produktstrukturen idealisiert auf und stellen gleichzeitig grundlegende Aufbauprinzipien der Produktstruktur dar. Sie sind nicht als sich gegenseitig ausschließende Alternativen zu verstehen, sondern können unternehmensspezifisch skaliert und miteinander kombiniert werden. 
In Bild 4 sind die Strategien für die Umsetzung modularer Produktstrukturen gegenübergestellt.

Gleichmodulstrategie (produktprogrammorientiert)

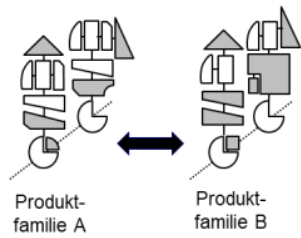

Modulbaukastenstrategie (variantenorientiert)

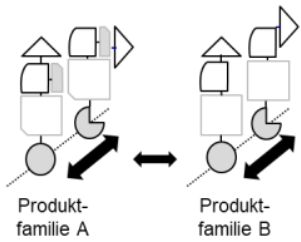

Plattformstrategie (produktfamilienorientiert)

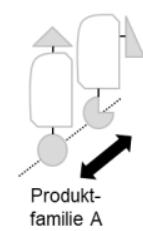

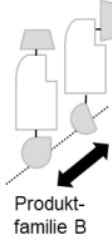

$\boldsymbol{\square}$ Variantenmodul produktfamilienübergreifend 口Standardmodul produktfamilienübergreifend

Variantenmodul in der Produktfamilie Standardmodul in der Produktfamilie

Bild 5: Gegenüberstellung der Gleichmodulstrategie, Modulbaukastenstrategie und Plattformstrategie. Abbildung in Anlehnung an [1, 2].

In Tabelle 2 sind verbreitete Strategien der Produktarchitekturgestaltung zusammenfassend dargestellt. Neben den beschriebenen Strategien zur Umsetzung modularer Produktstrukturen ist die Baureihenstrategie etabliert, um unterschiedliche Größenstufen von Komponenten oder Produkte zu realisieren.

Tabelle 2: Übersicht von Strategien zur Gestaltung der Produktarchitektur

\begin{tabular}{|l|l|}
\hline Strategie & Beschreibung \\
\hline $\begin{array}{l}\text { Gleich- } \\
\text { modul- } \\
\text { strategie }\end{array}$ & $\begin{array}{l}\text { Unter Gleichmodulstrategie (auch Mehrfachverwendung) von Modulen } \\
\text { wird die systematische Verwendung identischer Module über mehrere } \\
\text { Teile des Produktprogramms beziehungsweise im gesamten Produkt- } \\
\text { programm übergreifend in verschiedenen Produkten verstanden. Bei } \\
\text { zukünftigen Entwicklungsprojekten kann mithilfe der Gleichmodulstra- } \\
\text { tegie auf bereits vorhandene Module zurückgegriffen und dadurch Ent- } \\
\text { wicklungsaufwand und -risiko reduziert werden. }\end{array}$ \\
\hline $\begin{array}{l}\text { Modulbau- } \\
\text { kasten- } \\
\text { strategie }\end{array}$ & $\begin{array}{l}\text { Bei der Modulkastenstrategie wird ein Satz unterschiedlicher Module } \\
\text { genutzt, um Produktvarianten durch deren Kombination zu erzeugen. } \\
\text { Einzelne austauschbare Module werden als eigenständige Einheiten } \\
\text { gebildet, die sich stark an den Kundenbedürfnissen und deren Variatio- } \\
\text { nen ausrichten. Durch die Reduktion der Anzahl verwendeter Module } \\
\text { werden Stückzahleneffekte in einer Produktfamilie durch kommunale } \\
\text { Verwendung der Module erzielt. In neuen Produktgenerationen redu- } \\
\text { ziert die Übernahme von Modulen den Entwicklungsaufwand. }\end{array}$ \\
\hline
\end{tabular}


Tabelle 2: Übersicht von Strategien zur Gestaltung der Produktarchitektur (Fortsetzung)

\begin{tabular}{|l|l|}
\hline Strategie & Beschreibung \\
\hline $\begin{array}{l}\text { Plattform- } \\
\text { strategie }\end{array}$ & $\begin{array}{l}\text { Bei der Plattformstrategie wird eine Plattform als übergreifende, ge- } \\
\text { meinsame Basis innerhalb einer Produktfamilie definiert, um Pro- } \\
\text { duktvarianten abzuleiten und effizient produzieren zu können. In- } \\
\text { nerhalb der Plattform werden Module, die über alle Produktvarian- } \\
\text { ten einer Produktfamilie vereinheitlicht werden können und robust } \\
\text { gegenüber zukünftigen Änderungen der Kundenanforderungen an- } \\
\text { gesehen werden, zusammengefasst. Dadurch soll sichergestellt } \\
\text { werden, dass mit der standardisierten Plattform ein planbarer } \\
\text { Stückzahleneffekt erzielt wird. }\end{array}$ \\
\hline $\begin{array}{l}\text { Baureihen- } \\
\text { strategie }\end{array}$ & $\begin{array}{l}\text { Bei der Baureihenstrategie werden Produktvarianten durch Realisie- } \\
\text { rung unterschiedlicher Größenstufen realisiert. Die Produkte inner- } \\
\text { halb einer Baureihe sind hinsichtlich Funktion und prinzipieller Lö- } \\
\text { sung zur Erfüllung der Funktion bei allen Varianten gleich (Pahl and } \\
\text { Beitz, 1997). Durch die Realisierung der Lösung in unterschiedli- } \\
\text { chen Größenstufen können unterschiedliche Wertebereiche von Ei- } \\
\text { genschaften des Produkts realisiert werden, um effizient eine hohe } \\
\text { externe Produktvielfalt anbieten zu können. }\end{array}$ \\
\hline
\end{tabular}

\section{Methoden zur Gestaltung der Produktarchitektur}

Es existiert eine Vielzahl an Methoden zur Unterstützung der Gestaltung der Produktarchitektur. Diese Methoden geben ein planmäßiges Vorgehen und Hilfsmittel für die Umsetzung von Strategien und Realisierung von Bauweisen an. Die einzelnen Methoden fokussieren dabei unterschiedliche Zielstellungen. Für spezielle Entwicklungsprojekte stellt sich daher häufig die Frage, welche Methoden die Produktarchitekturgestaltung innerhalb des Produktentwicklungsprozesses unterstützen [7, 15]. Hilfestellungen für die Auswahl geeigneter Methoden können die skizzierten Zielstellungen, beispielsweise Reduzierung der Bauteileanzahl, Erhöhung der Änderbarkeit der Produkte oder Beherrschung von Variantenvielfalt geben. Weiterhin erfordern die einzelnen Methoden spezifische Informationen über das Produkt, beispielsweise Funktionsstrukturen oder prinzipielle Lösungen. Hierdurch ergibt sich ein unmittelbarer Bezug zu den Aktivitäten des Entwicklungsprojektes. Die nachfolgende Übersicht ausgewählter Methoden gibt eine erste Hilfestellung für die zweckmäßige Auswahl und Unterstützung der Produktarchitekturgestaltung. 
Tabelle 2: Übersicht ausgewählter Methoden für die Gestaltung der Produktarchitektur

\begin{tabular}{|l|l|l|}
\hline Methode & Quelle & Kurzbeschreibung \\
\hline $\begin{array}{l}\text { Systematisches } \\
\text { Vorgehen bei } \\
\text { der Funktionsin- } \\
\text { tegration }\end{array}$ & Roth [16] & $\begin{array}{l}\text { Identifizierung von Potentialen zur Funktions- } \\
\text { integration innerhalb von Baugruppen durch } \\
\text { Zusammenfassung von Wirkkörpern und Wirk- } \\
\text { flächen }\end{array}$ \\
\hline $\begin{array}{l}\text { Strategie der } \\
\text { einteiligen Ma- } \\
\text { schine }\end{array}$ & Ehrlenspiel [17] & $\begin{array}{l}\text { Identifizierung von Potentialen zur Reduktion } \\
\text { der Bauteilanzahl zur Einsparung von Herstell- } \\
\text { kosten durch Zusammenfassung von Bauteilen }\end{array}$ \\
\hline $\begin{array}{l}\text { Theory of Modu- } \\
\text { lar Design }\end{array}$ & Stone [18] & $\begin{array}{l}\text { Modularisierung des Produkts durch Heuristi- } \\
\text { ken auf Basis der Funktionsstruktur }\end{array}$ \\
\hline $\begin{array}{l}\text { Integration Ana- } \\
\text { lysis Methodo- } \\
\text { logy }\end{array}$ & $\begin{array}{l}\text { Steward [19], } \\
\text { Pimmler und } \\
\text { Eppinger [20] }\end{array}$ & $\begin{array}{l}\text { Modularisierung des Produkts zur Reduzierung } \\
\text { der Produktkomplexität auf Basis von funktio- } \\
\text { nalen und physischen Beziehungen zwischen } \\
\text { Komponenten }\end{array}$ \\
\hline $\begin{array}{l}\text { Vorgehen beim } \\
\text { Entwickeln von } \\
\text { Baukästen }\end{array}$ & $\begin{array}{l}\text { Pahl und Beitz } \\
{[11]}\end{array}$ & $\begin{array}{l}\text { Entwicklung einer Baukastensystematik für } \\
\text { Produktfamilien auf Basis von Varianz der Teil- } \\
\text { funktionen }\end{array}$ \\
\hline $\begin{array}{l}\text { Modular Func- } \\
\text { tion Deployment }\end{array}$ & Erixon [21] & $\begin{array}{l}\text { Modularisierung des Produkts durch Analyse } \\
\text { der Auswirkungen strategischer Modultreiber } \\
\text { auf Komponenten des Produkts }\end{array}$ \\
\hline $\begin{array}{l}\text { Product Family } \\
\text { Master Plan }\end{array}$ & $\begin{array}{l}\text { Mortensen [22], } \\
\text { Harlou [23] }\end{array}$ & $\begin{array}{l}\text { Modellierung und Handhabung der Varianten- } \\
\text { vielfalt innerhalb von Produktfamilien }\end{array}$ \\
\hline $\begin{array}{l}\text { Integrierter } \\
\text { PKT-Ansatz zur } \\
\text { Entwicklung mo- } \\
\text { dularer Produkt- } \\
\text { familien }\end{array}$ & $\begin{array}{l}\text { Krause und } \\
\text { Gebhardt [2] }\end{array}$ & $\begin{array}{l}\text { Entwicklung modularer Produktfamilien unter } \\
\text { Berücksichtigung technisch-funktionaler und } \\
\text { produktstrategischer Aspekte zur Reduzierung } \\
\text { der internen Varianz }\end{array}$ \\
\hline
\end{tabular}

\section{$7 \quad$ Forschungsfelder der Produktarchitekturgestaltung}

Die eingeführten Begriffe und beschriebenen Zielstellungen, Bauweisen, Strategien und Methoden sollen eine zielgerichtete Gestaltung der Produktarchitektur unterstützen und die Vermittlung wesentlicher Zusammenhänge vereinfachen. Die vorgenommene Strukturierung und Beschreibung basiert auf etablierten Forschungsarbeiten und stellt damit eine gute Wissensbasis dar. Um 
die Produktarchitekturgestaltung in der Produktentwicklung weiter zu unterstützen und mit anderen Entwicklungsaktivitäten zu vernetzen, ergeben sich unter anderem folgende Forschungsfelder. Basierend auf Ansätzen der modellbasierten Entwicklung sollen Konzepte für die modellbasierte Abbildung, Analyse und Evolution von Produktarchitekturen erarbeitet werden. Im Vordergrund stehen hierbei die Sicherstellung einer durchgängigen Modellierung sowie die enge Verknüpfung von Prozess- und Produktinformationen [24]. Ein weiteres Forschungsfeld stellt die Integration der Produktarchitekturgestaltung in den Entwicklungsprozess dar. Hierbei sind erforderliche Analyse-, Gestaltungs- sowie -aktivitäten und -ergebnisse mit den übrigen Entwicklungsaktivitäten abzugleichen und in Abhängigkeit der verfolgten Zielstellungen in geeignete Konkretisierungsebenen zu integrieren. Diese Einbettung der Produktarchitekturgestaltung kann durch ein allgemeingültiges Vorgehen unterstützt werden. Um das Vorgehen zu strukturieren existieren zwar einzelne Ansätze, siehe [2] diese sind allerdings auf spezifische Zielstellungen ausgerichtet, weshalb ihre Allgemeingültigkeit zu überprüfen ist. Weiterhin ist die Einordnung geeigneter Methoden anzustreben. Um die Handhabung der zunehmend heterogenen Lebenszyklen in technischen Systemen durch eine geeignete Produktarchitekturgestaltung zu unterstützen, ergibt sich als weiteres Feld zukünftiger Forschungsarbeiten die Modellierung der heterogenen Lebensdauern in komplexen Systemen. Auf Basis der maßgeblichen Treiber der Lebensdauern einzelner Komponenten soll die Definition geeigneter Lebenszyklusoptionen und deren Umsetzung durch eine geeignete Produktarchitektur unterstützt werden [25].

\section{Zusammenfassung}

Ziel dieses Beitrages war es, die Relevanz der Produktarchitektur als zentrales Konzept in der Produktentstehung zu verdeutlichen. Um die bestehende Konzepte und Ansätze für die Produktarchitekturgestaltung anzugleichen und zu systematisieren, wurden zentrale Begriffe und deren Zusammenhänge beschrieben. Die Gestaltung der Produktarchitektur erfolgt demnach ausgehend von unterschiedlichen Zielstellungen, die sowohl Produkteigenschaften als auch Prozesse betreffen und aus Kunden- und Unternehmenssicht relevant sind. Ergebnis der zielgerichteten Gestaltung der Produktarchitektur sind unterschiedliche Bauweisen, die in Form von Konstruktionsprinzipien formuliert und bereitgestellt werden können. Strategien beispielsweise zur Beherrschung der Variantenvielfalt, wie die Verwendung einer modularen Produktstrukturstrategie, sind hierbei häufig maßgeblich und sowohl technisch-funktionale als auch produktstrategische Aspekte zu berücksichtigen. Als Hilfestellung für die Identifizierung von Bauweisen und Strategien sind unterschiedliche Methoden verfüg- 
bar, die die Erreichung unterschiedlicher Zielstellungen und die hierfür erforderlichen Analyse- und Syntheseaktivitäten unterstützen können. In diesem Beitrag wurden exemplarische Zielstellungen und etablierte Bauweisen, Strategien und ausgewählte Methoden für die Gestaltung der Produktarchitektur aufgezeigt. Ausgehend von der eingeführten Strukturierung der Begriffe wurden im Beitrag mögliche Forschungsfelder im Zusammenhang mit der Produktarchitekturgestaltung skizziert.

\section{Anmerkung}

Die in diesem Beitrag beschriebenen Inhalte stellen Auszüge des Buchkapitels Produktarchitektur der neunten Auflage des Lehrbuchs Pahl/Beitz Konstruktionslehre [5] dar.

\section{Literatur}

[1] Krause, D. et al.: "Modulare Produktstrukturierung", In: Steinhilper, F.R.R. (Ed.), Handbuch Konstruktion, pp. 719-741. Hanser Verlag, München, 2018.

[2] Krause, D.; Gebhardt, N.: "Methodische Entwicklung modularer Produktfamilien: Hohe Produktvielfalt beherrschbar entwickeln", Springer-Verlag, Heidelberg, 2018.

[3] Ulrich, K. T.; Tung, K.: "Fundamentals of Product Modularity" Sloan School of Management, Massachusetts Institute of Technology, 1991.

[4] Ulrich, K. T.: "The role of product architecture in the manufacturing firm", Research Policy, Vol 24, No 3, pp. 419-440, 1995.

[5] Krause, D. et al.: "Produktarchitektur", In: Bender, B.; Gericke, K.: "Pahl/Beitz Konstruktionslehre - Methoden und Anwendung erfolgreicher Produktentwicklung", Springer-Verlag, Heidelberg, 2020

[6] Yassine, A. A., Wissmann, L. A.: "The Implications of Product Architecture on the Firm", Systems Engineering Vol 10, No 2, pp. 118-137, 2007.

[7] Bonvoisin, J. et al.: "A systematic literature review on modular product design", Journal of Engineering Design, Vol 27, No 7, pp. 488-514, 2016.

[8] Franke, H.-J., Schill, J.: "Kosten senken durch Einsparen von Teilen", pp. 139$152,1987$.

[9] Vietor, T.; Herrmann, C.; Spengler, T.: "Synergetische Produktentwicklung - Unternehmensübergreifend erfolgreich zusammenarbeiten. Ergebnisse des Verbundprojekts SynProd". Shaker-Verlag, Aachen, 2015.

[10] Franke, H.-J. et al.: "Variantenmanagement in der Einzel- und Kleinserienfertigung", Carl Hanser Verlag, München, 2002. 
[11] Pahl, G.; Beitz, W.: "Konstruktionslehre: Methoden und Anwendung", SpringerVerlag, Heidelberg, 1997.

[12] Ziebart, J. R.: "Ein konstruktionsmethodischer Ansatz zur Funktionsintegration", Dissertation, TU Braunschweig, 2012.

[13] Klein, B.: "Leichtbau-Konstruktion: Berechnungsgrundlagen und Gestaltung", Springer-Verlag, Heidelberg, 2013.

[14] Koller, R.: "Konstruktionslehre für den Maschinenbau - Grundlagen zur Neu- und Weiterentwicklung technischer Produkte mit Beispielen", Springer-Verlag, Heidelberg, 1994.

[15] Otto, K. et al.: "Global Views on Modular Design Research: Linking Alternative Methods to Support Modular Product Family Concept Development", Journal of Mechanical Design, Vol 138, No 7, pp. 071101.1-071101.16, 2016.

[16] Roth, K.: "Konstruieren mit Konstruktionskatalogen - Band 1: Konstruktionslehre", Springer-Verlag, Heidelberg, Berlin, New York, 2000.

[17] Ehrlenspiel, K.: "Kostengünstig Konstruieren: Kostenwissen, Kosteneinflüsse, Kostensenkung", Springer-Verlag, Heidelberg, Berlin, New York, 1995.

[18] Stone, R. B.: "Towards a theory of modular design", Dissertation, University of Texas, 1997.

[19] Steward, D. V.: "The design structure system: A method for managing the design of complex systems", IEEE Transactions on Engineering Management, Vol 28, No 3, pp. 71-74, 1981.

[20] Pimmler, T. U.; Eppinger, S. D.: "Integration analysis of product decompositions", Proceedings ASME 1994 International Design Engineering Technical Conferences \& 6th Design Theory and Methodology Conference, Minneapolis, Minnesota, pp. 343-351, 1994.

[21] Erixon, G.: "Modular function deployment: a method for product modularisation", Dissertation, Royal Inst. of Technology, 1998.

[22] Mortensen, N. H.: "Design modelling in a Designer's Workbench--Contribution to a Design Language", Dissertation, Technical University of Denmark, 1999.

[23] Harlou, U.: "Developing product families based on architectures: Contribution to a theory of product families", Dissertation, Technical University of Denmark, 2006

[24] Inkermann, D.: "Towards Model-Based Process Engineering", Proceedings International Conference on Engineering Design - ICED 2019, Delft, Niederlande, pp. 3741-3750, 2019.

[25] Umeda, Y.; Daimon, T.; Kondoh, S.: "Life Cycle Option Selection based on the Difference of Value and Physical Lifetimes for Life Cycle Design", Proceedings International Conference on Engineering Design - ICED 2007, Paris, France, Paper No. DS42_P_474, 2007. 